\title{
BLOWUP OF NONLINEAR SCHRÖDINGER EQUATIONS WITH INVERSE-SQUARE POTENTIALS
}

\author{
TOSHIYUKI SUZUKI
}

Abstract. Blowup in finite time for nonlinear Schrödinger equations (NLS) with inverse-square potential $a|x|^{-2}$ and nonlocal nonlinearities described by integral operators (HE) ${ }_{a}$ is considered. The local and global existence for (HE) ${ }_{a}$ is studied in Suzuki [10]. To show the blowup for (NLS) the virial identity is important role. But the identity for (HE) ${ }_{a}$ has not proved in consequence of the strongly singular potential. Thus we give a strict proof of the virial identity for (HE) $)_{a}$.

Mathematics subject classification (2010): 35Q55, 35Q40, 81Q15.

Keywords and phrases: blowup, Hartree equations, nonlinear Schrödinger equations, virial identity, inverse-square potentials.

\section{REFERENCES}

[1] H. Berestycki, T. CaZenave, Instabilité des états stationaires dans les équations de Schrödinger et de Klein-Gordon non linéaires, C. R. Acad. Sci. Paris Sér. I Math., 293 (1981), 489-492.

[2] N. Burq, F. Planchon, J. Stalker, A. S. TAhVildar-Zadeh, Strichartz estimates for the wave and Schrödinger equations with the inverse-square potential, J. Funct. Anal., 203 (2003), 519-549.

[3] T. Cazenave, "Semilinear Schrödinger Equations," Courant Lecture Notes in Mathematics, 10. New York University, Courant Institute of Mathematical Sciences, New York; American Mathematical Society, 2003.

[4] J. M. Chadam, R. T. Glassey, Global existence of solutions to the Cauchy problem for timedependent Hartree equations, J. Math. Phys., 16 (1975), 1122-1130.

[5] J. Ginibre, G. Velo, On a class of nonlinear Schrödinger equations with nonlocal interaction, Math. Z., 170 (1980), 109-136.

[6] R. T. Glassey, On the blowing up of solutions to the Cauchy problem for nonlinear Schrödinger equations, J. Math. Phys., 18 (1977), 1794-1797.

[7] E. H. Lieb, B. Simon, The Hartree-Fock theory for Coulomb systems, Comm. Math. Phys., 53 (1977), 185-194.

[8] T. OgAwa, Y. Tsutsumi, Blow-up of $H^{1}$-solution for the nonlinear Schrödinger equation, J. Differential Equations, 92 (1991), 317-330.

[9] N. OKAZAWA, T. SUZUKI, T. YокотA, Energy methods for abstract nonlinear Schrödinger equations, Evol. Equ. Control Theory, 1 (2012), 337-354.

[10] T. SUZUKI, Energy methods for Hartree type equation with inverse-square potentials, Evol. Equ. Control Theory, 2 (2013), 531-542.

[11] M. Weinstein, Nonlinear Schrödinger equations and sharp interpolation estimates, Comm. Math. Phys., 87 (1982), 567-576. 